\title{
Adaptive Use of Information during Growth Can Explain Long-Term Effects of Early Life Experiences
}

\author{
Sinead English, ${ }^{1,2, \star}$ Tim W. Fawcett, ${ }^{3,4}$ Andrew D. Higginson, ${ }^{3,4}$ Pete C. Trimmer, ${ }^{3,5}$ \\ and Tobias Uller ${ }^{1,6}$ \\ 1. Edward Grey Institute, Department of Zoology, University of Oxford, South Parks Road, Oxford OX1 3PS, United Kingdom; \\ 2. Behavioural Ecology Group, Department of Zoology, University of Cambridge, Downing Street, Cambridge CB2 3EJ, United King- \\ dom; 3. Modelling Animal Decisions Group, School of Biological Sciences, University of Bristol, Woodland Road, Bristol BS8 1UG, \\ United Kingdom; 4. Centre for Research in Animal Behaviour, Washington Singer Laboratories, University of Exeter, Exeter EX4 \\ 4QG, United Kingdom; 5. Department of Environmental Science and Policy, University of California at Davis, One Shields Avenue, \\ Davis, California 95616; 6. Department of Biology, Lund University, Sölvegatan 37, Lund SE 223 62, Sweden
}

Submitted August 19, 2015; Accepted December 4, 2015; Electronically published March 15, 2016

Online enhancements: appendixes.

A Bstract: Development is a continuous process during which individuals gain information about their environment and adjust their phenotype accordingly. In many natural systems, individuals are particularly sensitive to early life experiences, even in the absence of later constraints on plasticity. Recent models have highlighted how the adaptive use of information can explain age-dependent plasticity. These models assume that information gain and phenotypic adjustments either cannot occur simultaneously or are completely independent. This assumption is not valid in the context of growth, where finding food results both in a size increase and learning about food availability. Here, we describe a simple model of growth to provide proof of principle that long-term effects of early life experiences can arise through the coupled dynamics of information acquisition and phenotypic change in the absence of direct constraints on plasticity. The increase in reproductive value from gaining information and sensitivity of behavior to experiences declines across development. Early life experiences have longterm impacts on age of maturity, yet - due to compensatory changes in behavior - our model predicts no substantial effects on reproductive success. We discuss how the evolution of sensitive windows can be explained by experiences having short-term effects on informational and phenotypic states, which generate long-term effects on life-history decisions.

Keywords: information use, development, state dependence, plasticity, sensitive periods, Bayesian updating.

\section{Introduction}

Development is a continuous, constructive process during which individuals may be capable of responding to the environment by adjusting their phenotype to local conditions (West-Eberhard 2003; Frankenhuis and Panchanathan 2011a).

* Corresponding author; e-mail: sineadenglish@cantab.net.

Am. Nat. 2016. Vol. 187, pp. 620-632. (C) 2016 by The University of Chicago. 0003-0147/2016/18705-56474\$15.00. All rights reserved.

DOI: $10.1086 / 685644$
Although, in principle, organisms might have the potential to respond to the environment throughout development, they often exhibit sensitive windows, which are periodstypically early in life-during which the phenotypic effects of the environment are particularly marked (Bateson 1979; Lindström 1999; Fawcett and Frankenhuis 2015). Classic examples of sensitive windows include imprinting of sexual preferences in birds (Bateson 1966), long-term effects of early hormone exposure on behavior (Kaiser and Sachser 2005; Ellis et al. 2011), early growth conditions affecting life history in wild vertebrates (e.g., salmonids [Metcalfe et al. 1988], ungulates [Albon et al. 1987; Plard et al. 2015], birds [Cam et al. 2003]), and fetal programming of cognitive and physical health in humans (Lucas 1998).

An intuitive explanation for sensitive windows is that there are constraints on an individual's ability to adjust its phenotype later in life (Dufty et al. 2002), as seen in organisms with discrete life stages where, for example, adjustments after metamorphosis are not possible (Whitman and Agrawal 2009). However, differential sensitivity to the environment across development often occurs in the absence of constraints on plasticity (Bateson 1979; Hoverman and Relyea 2007). This raises the question of whether long-term effects of early life experiences can evolve in the absence of such constraints. One intriguing possibility is that sensitive windows arise from adaptive developmental responses to the environment, through changes in an individual's informational and physiological states (Dufty et al. 2002; Frankenhuis and Panchanathan 2011a). Experiences of the environment during development can have immediate effects on an individual's state - for example, its size, which affects statedependent behavior - and in so doing may feed back to influence future state (fig. 1). For example, larger individuals may be less vulnerable to predation and so spend a greater pro- 


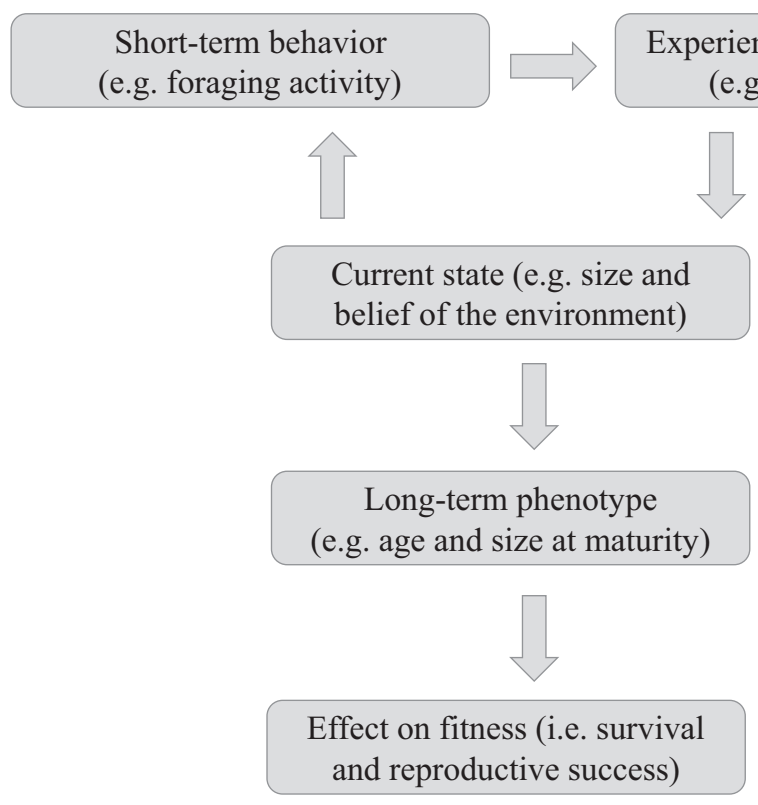

Figure 1: Pathways by which experiences of the environment can have long-term effects on fitness. Arrows indicate the direction of causation.

portion of time foraging, enabling them to become even larger still (Werner 1986). These experiences may have only small immediate effects but substantial delayed or carryover impacts at later life stages (Harrison et al. 2011).

Long-term effects of early experiences may also arise if information is more valuable to individuals at an early stage, when a greater range of potential developmental trajectories and, hence, phenotypic outcomes are available (Dufty et al. 2002). In this sense, offspring might be adaptively primed by early life information, from their parents or from direct interactions with the environment, to produce a phenotype best matched to the conditions they are likely to experience later in life (Gluckman et al. 2005a; Worthman and Kuzara 2005; Uller 2008). Such predictive adaptive responses are contingent on parental or early life cues reliably predicting the later environment (Burgess and Marshall 2014): in cases where there is a mismatch between the predicted and realized environments, an inappropriate phenotype is developed and individuals may suffer a fitness cost (Gluckman et al. 2005b). Thus, the strongest evidence for such adaptive plasticity has been found in species with a short life cycle relative to the rate of environmental change (e.g., Daphnia [Agrawal et al. 1999] and the butterfly Bicyclus anynana [Saastamoinen et al. 2010]) where individuals in successive generations are likely to encounter similar conditions.

In spite of the growing empirical evidence of the longterm effects of early life experiences, there have-until recently-been few attempts to develop theoretical models on how such effects might evolve. In recent years, a collec- tion of models have considered the adaptive use of information during development and its role in generating agedependent patterns of plasticity (reviewed in Fawcett and Frankenhuis 2015). These models have demonstrated that heightened plasticity early in life can arise if environmental cues do not reliably indicate the environmental state-resulting in uncertainty about the most suitable phenotypeand if phenotypic adjustments entail physiological costs (Fischer et al. 2014) or opportunity costs, for example, when developing the appropriate phenotype for one environmental state reduces the time available for investing in alternative phenotypes suited to other states (Frankenhuis and Panchanathan 2011a). Moreover, Frankenhuis and Panchanathan $(2011 a, 2011 b)$ have shown that the duration of such sensitive windows may vary among individuals depending on experience: those who receive a more consistent set of cues about their environment lose sensitivity more rapidly.

Many empirical examples of the long-term effects of early conditions focus on aspects of growth and life history, such as age and size at maturation (Kuzawa et al. 2010; Lee et al. 2012; Douhard et al. 2013; Lee and Bussière 2013). It is important to recognize that information gain and phenotypic adjustment are tightly coupled during growth (Stamps and Krishnan 2014) because food consumption results in both an increase in size and learning about food availability. Existing models of adaptive developmental plasticity (Frankenhuis and Panchanathan 2011a; Fischer et al. 2014), however, have considered information gain and phenotypic adjustment either as mutually exclusive options or as independent 
processes. There is a rich body of theoretical work on adaptive growth trajectories (Case 1978), in which some models have examined the link between early conditions, growth, and later life history. These models have primarily focused on salmonid life history (Thorpe et al. 1998; Satterthwaite et al. 2009, 2010) and assume that individuals use their recent growth rate during an assumed fixed window as a cue in making life-history decisions. These growth models do not, however, explicitly address how continuous sampling of the environment across development can generate the evolution of sensitive windows at particular developmental stages. New theory is therefore needed to draw a link between these modeling approaches - on the continuous use of information, on the one hand, and on the process of growth, on the other - and assess whether the adaptive use of information during growth can generate sensitive windows in the absence of constraints on plasticity.

Here, we present a generally applicable proof-of-concept model demonstrating how the use of information during growth before sexual maturity can generate sensitive windows in development. In our model, individuals decide at each time step whether to mature or continue growing based on their size and their estimation of predation risk and food availability in their environment. We use Bayesian updating as a framework to model how individuals accrue information based on their own experiences and input from previous generations (Frankenhuis and Panchanathan 2011a; Fischer et al. 2014; Stamps and Krishnan 2014). Outcomes of behavioral decisions (e.g., discovering food after a certain amount of time spent foraging) have a direct, coupled effect on an individual's phenotypic state (e.g., increase in size) and information state (e.g., increase in the probability that food is abundant). We quantify how the value of information changes across development by considering the difference in fitness when individuals gain information compared to when they do not gain information (McNamara and Dall 2010; Lister 2014). We run simulation experiments to examine the effects of varying the accuracy of cues received at the start of life (which could represent genetic or parental information about conditions; Leimar et al. 2006; Shea et al. 2011; Stamps and Krishnan 2014) and of being exposed to temporary food supplementation or deprivation at different points in development on phenotypic outcome and reproductive success. Conducting these initial-cue and food-manipulation experiments in different environments allows us to explore the phenotypic and fitness consequences of experiences during growth that are matched or mismatched to the prevailing environment.

\section{The Model}

We consider a world comprising discrete patches that have high or low food availability and that differ in preda- tion pressure (see below). A schematic diagram describing our model is given in figure 2 . We assume a life history in which - following dispersal to a randomly selected patch after conception-individuals develop, reproduce, and die in the same patch. At each time step in development, individuals decide whether to stop growing and commence reproduction (i.e., mature) or to continue foraging in order to grow. If the latter, individuals also decide how much to forage. If an individual dies prior to reproduction, its realized reproductive value $(V)$ is zero. Otherwise, realized reproductive value is a sigmoidal function of the individual's size $(S)$ at the onset of reproduction and depends on the environmental state ( $E_{i}$; app. A; fig. $2 B$; apps. A-C available online). Thus, our model describes growth until maturity and assumes that reproductive success is a function of both size at maturity and environmental conditions. This assumption can apply to both semelparous and iteroparous organisms but may not hold for species in which individuals continue to grow after they reach sexual maturity (i.e., indeterminate growth). We assume random natal dispersal as this allows us to focus on the case where individuals start life with maximum uncertainty about their environment. Our model is most relevant to organisms with low levels of dispersal during development or to scenarios where the rate of environmental change is much lower than the rate at which organisms encounter new environments.

While reproductive success increases with size at maturity in both environments, individuals in the high-food environment $\left(E_{1}\right)$ benefit more from reaching a larger size, and small individuals have higher reproductive success in the low-food environment $\left(E_{2}\right)$ than in the high-food environment (fig. $2 B$ ). We chose this relationship between size and reproduction to reflect two environment-specific differences in the costs and benefits of large body size. First, reproductive competition may be more intense in highfood environments - for example, if individuals have more time and energy to spend on competing for mates - thus individuals in these environments benefit more from having a large body size than they would in low-food environments. Second, the low food availability in low-food environments might impose a higher cost of maintaining large body size than it would in high-food environments, meaning it is better to be small (Jönsson 1997).

If not maturing, individuals choose the proportion of the next time step to spend actively foraging, $\alpha(0 \leq \alpha \leq 1)$. For a given level of foraging effort, individuals are more likely to be successful at discovering food in the high-food environment than in the low-food environment (fig. 2C):

$$
\operatorname{Pr}\left(\operatorname{succ} \mid E_{i}, \alpha\right)=\alpha \beta_{i} \quad i=1,2,
$$

where $\operatorname{Pr}($ succ) denotes the probability that an individual is successful at finding a food item, and $\beta_{i}\left(\beta_{1}>\beta_{2}\right)$ is the 


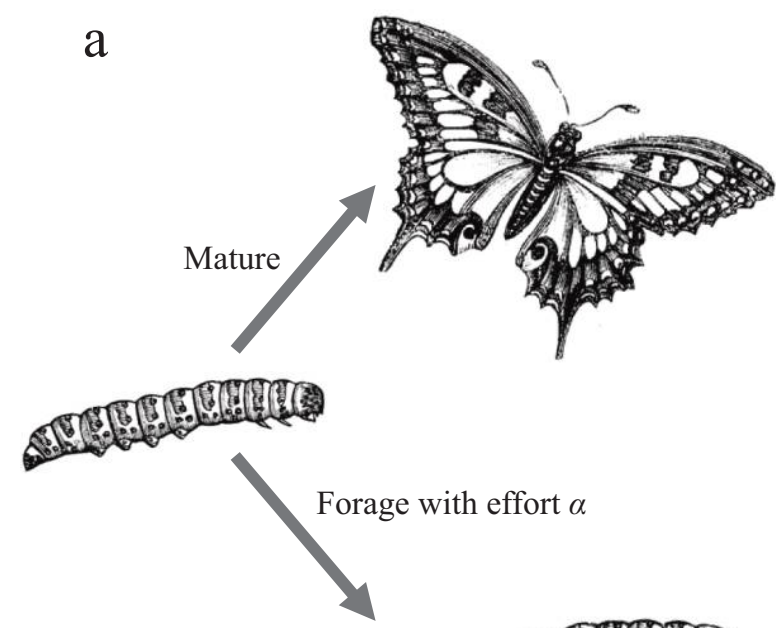

State at $t=(S, P)$

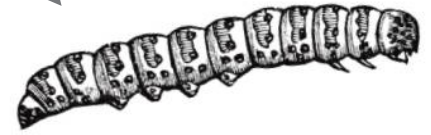

State at $t+1=\left(S^{\prime}, P^{\prime}\right)$

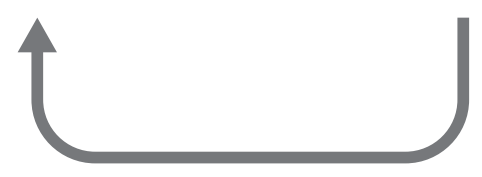

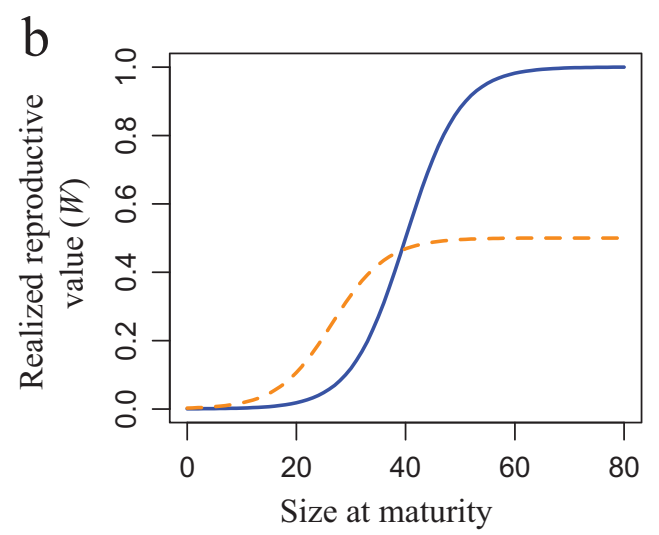

C
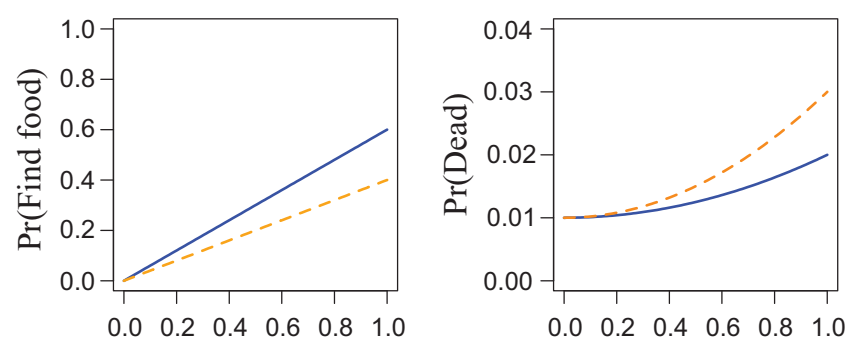

Foraging effort $(\alpha)$

Figure 2: Schematic diagram outlining the model setup and describing the reproduction, growth, and mortality functions. $a$, Life-history decisions faced by the model organism at each time step (whether to mature and reproduce or forage and continue growing) depending on its state (size, $S$, and belief, $P$ ). $b$, Relationship between size at maturity and realized reproductive value in the high-food (blue, solid line) and low-food (orange, dashed line) environments. Relationship between probability of finding food (c) or probability of being predated (d) depending on foraging effort in the high-food (blue, solid line) and low-food (orange, dashed line) environments. Note that we select a holometabolous insect as an example model, but our model is a general one and can apply to other scenarios such as sessile marine invertebrates with pelagic eggs. Images: butterfly larva, from J. G. Wood, Third Natural History Reader (Boston School Supply, Boston, 1896, p. 182, retrieved from http://etc.usf.edu/clipart/3100/3125/grub_1.htm); butterfly adult, from Worthington Hooker, The Child's Book of Nature (Harper, New York, 1886, p. 47, retrieved from http://etc.usf.edu/clipart/3200/3236/butterfly_3.htm).

probability of finding food at the maximum foraging effort (i.e., when $\alpha=1$ ). If a food item is found, size $(S)$ increases by one unit; that is, size increases linearly with food consumption. We modeled growth in this simplified manner in order to examine general principles; more complex formulations of growth (e.g., a nonlinear decreasing function with age) may lead to more detailed predictions tailored to particular life histories.

The probability of mortality per time step, $\mu$, increases as an accelerating function of $\alpha$, the slope of which depends on the environment (fig. 2D):

$$
\mu\left(\alpha, E_{i}\right)=\mu_{0}\left(1+\mu_{i} \alpha^{\gamma}\right) \quad i=1,2,
$$

where $\mu_{0}$ is the baseline mortality at zero foraging effort (constant across environments), $\mu_{i}$ is the environment-specific increase in mortality, and $\gamma$ controls the shape of the relationship between foraging effort and mortality $(\gamma>1)$. Here, in the main text, we consider a scenario where foraging entails lower risks in the high-food environment $\left(\mu_{1}<\mu_{2}\right)$. Such coupling of predation risk and food availability could occur when high-food environments have high vegetation cover, thus providing more food and more shelter from predators. In appendix A, we consider two additional scenarios: one with constant predation risk across environments $\left(\mu_{1}=\mu_{2}\right)$, and one where individuals in the high-food environment suffer higher risk of predation $\left(\mu_{1}>\mu_{2}\right)$. This latter situation can arise when more productive environments support higher densities of predators. See table 1 for a summary of all model parameters and baseline values.

We use stochastic dynamic programming (Houston and McNamara 1999; Clark and Mangel 2000) to identify op- 
Table 1: Description of parameters and values used in the model

\begin{tabular}{|c|c|c|}
\hline Variable & Description & Value \\
\hline$\alpha$ & Level of foraging effort & 0-1 (501 levels) \\
\hline$S$ & Body size & $1-80$ \\
\hline$P$ & Belief of being in environment 1 & $0-1$ (501 levels) \\
\hline$\lambda_{i}$ & $\begin{array}{l}\text { Asymptotic reproductive value at } \\
\text { maturity (environment specific) }\end{array}$ & $\{1,2\}$ \\
\hline$\kappa_{i}$ & $\begin{array}{l}\text { Size at maturity at which half of } \\
\text { max. reproductive value is } \\
\text { achieved (environment specific) }\end{array}$ & $\{40,30\}$ \\
\hline$\phi$ & $\begin{array}{l}\text { Slope of reproductive value func- } \\
\text { tion at point of inflection }\left(\kappa_{i}\right)\end{array}$ & .2 \\
\hline$\beta_{i}$ & $\begin{array}{l}\text { Probability of finding food per } \\
\text { time step (environment specific) }\end{array}$ & $\{.6, .4\}$ \\
\hline$\mu_{0}$ & $\begin{array}{l}\text { Baseline probability of mortality } \\
\text { per time step }\end{array}$ & .01 \\
\hline$\mu_{i}$ & $\begin{array}{l}\text { Environment-specific mortality } \\
\text { parameter, depending on } \\
\text { scenario }\end{array}$ & $\{1,1\} ;\{1,2\} ;\{2,1\}$ \\
\hline$\gamma$ & $\begin{array}{c}\text { Power governing the increase in } \\
\text { mortality with foraging effort }\end{array}$ & 2 \\
\hline
\end{tabular}

timal decisions (whether to mature and how much to forage), which maximize reproductive value given current state. This framework provides a natural way to integrate the dynamic course of development with the optimization principles of evolutionary biology (Frankenhuis et al. 2013). In our model, an individual's state has two components: size, $S$, and an estimated probability (belief), $P$, that it is in the high-food environment. Belief $P$ is updated throughout development in a Bayesian manner, whereby at each time step, an individual's updated belief depends on both the new information sampled and its previous belief (for further explanation, see McNamara and Houston 1987; Mangel 1990; Hilborn and Mangel 1997; Clark and Mangel 2000; McNamara et al. 2006; Valone 2006; Trimmer et al. 2011; for similar models treating information as a state variable, see Mangel and Roitberg 1989). Information is thus based on cumulative experience. We explore the impact of an initial belief at the start of development, such as when information is inherited through genes or maternal effects (Leimar et al. 2006; McNamara and Dall 2010; Shea et al. 2011; Stamps and Krishnan 2014; English et al. 2015). Details of the dynamic optimization and Bayesian updating equations are provided in appendix A. Note that our approach operates through value iteration: age at maturity emerges from statedependent decisions rather than it being assumed that individuals must mature within a fixed number of time steps.

For decisions before maturity, we measure the benefit of using information from foraging experiences to adjust optimal foraging effort. This value of information $\left(V_{\text {INFO }}\right)$ is calculated as the difference between the reproductive value for a given state and the reproductive value for the same state but when behavior has not been updated according to experience (for a similar approach, see McNamara and Dall 2010). Specifically, for a given size and information state $\left\{S_{0}, P_{0}\right\}$ :

$$
\begin{aligned}
V_{\mathrm{INFO}}\left\{S_{0}, P_{0}\right\}= & \operatorname{Pr}(\text { surv }, \text { succ })\left[V\left(S_{0}, P_{+}, \alpha_{S_{0}, P_{+}}^{*}\right)\right. \\
& \left.-V\left(S_{0}, P_{+}, \alpha_{S_{0}, P_{0}}^{*}\right)\right] \\
& +\operatorname{Pr}(\text { surv, fail })\left[V\left(S_{0}, P_{-}, \alpha_{S_{0}, P_{-}}^{*}\right)\right. \\
& \left.-V\left(S_{0}, P_{-}, \alpha_{S_{0}, P_{0}}^{*}\right)\right]
\end{aligned}
$$

where $\operatorname{Pr}$ (surv, succ) and $\operatorname{Pr}($ surv, fail) denote the probability that an individual survives and succeeds in finding food or survives and fails to find food, respectively; subscript plus and minus signs denote values for updated belief $(P)$ if an individual finds food or fails to find food, respectively; and $\alpha_{S, P}^{*}$ is the level of foraging effort that optimizes expected reproductive value for individuals of size $S$ and belief $P$ (see app. A). Note that we do not use $S_{0}+1$ in the first term of the right-hand side of equation (3) in order to separate the value of information (current estimate, $P$ ) from the value of growth (and learning about the environment through having survived; Welton et al. 2003).

We then run three simulations to explore how foraging experiences shape development. First, we run a baseline simulation where individuals are born with maximum uncertainty of being in the high-food or low-food environment, that is, a prior probability of being in the high-food environment (hereafter, starting belief) of $P=0.5$. Second, we investigate the importance of information at the start of the growth period using simulations in which the starting belief is $P=0.1$ or $P=0.9$. Third, we investigate sensitivity to the environmental conditions across development using simulations in which the probability of food encounter is fixed at 0.1 (temporary deprivation) or 0.9 (temporary supplementation) for a period of 10 time steps, starting at $0,10,20,30,40$, or 50 time steps into development. We select these windows such that they occur before the age of maturity (maturity being based on state rather than being time limited, as explained above) and take up a substantial fraction (about 10\%) of an individual's development. We conduct all manipulations in both environments, thus allowing us to investigate the effect of starting beliefs or experiences that are matched (e.g., $P=0.1$, or temporary deprivation in the low-food environment) or mismatched (e.g., $P=0.9$, or temporary supplementation in the low-food environment) to the environment. For all simulations, individuals are initialized with a size of $S=1$ at birth. Each simulation is run using 10,000 individuals. Fitness is measured by multiplying the proportion of individuals that survive to maturity with the mean reproductive value of survivors (depending both on size at maturity and 
the environment; fig. $2 B$ ). To visualize changes in state and the value of information across age, we repeat the simulations without mortality for 100 individuals. The model was written in the $\mathrm{C}++$ programming language, and the simulation data were processed and plotted using R ( R Development Core Team 2013). All scripts and simulation data are available online at Github: http://dx.doi.org/10.5281/zenodo .35322 (English 2015).

\section{Results}

The optimal size at maturity increases as individuals become more certain that they are in the high-food environment (i.e., as $P$ increases), and the optimal foraging effort $\left(\alpha^{*}\right)$ is higher in the environment with lower predation (figs. $3 a, \mathrm{~A} 1 a$, A1d; figs. A1-A4 available online). For comparison, we also derive an analytical solution for the optimal foraging effort and size at maturity for an individual that has perfect knowledge of the environmental state, based on the assumptions that behavior is optimized to maximize the growth-to-mortality ratio (Werner and Gilliam 1984) and that individuals mature when the reproductive value from doing so outweighs that from continuing to grow (see details in app. B). The analytical predictions closely match those from the dynamic optimization in the case where individuals have perfect knowledge of the environmental state ( $P=0$ or $P=1$; app. B). Simulated developmental trajec- tories for individuals starting life with a uniform prior $(P=0.5)$ demonstrate that most individuals learn, rapidly and correctly, which environment they are in and mature at the appropriate size (fig. A2).

Both the value of information and the sensitivity of behavior to particular foraging experiences are greater earlier in life and decline gradually across development (fig. $3 b$, $3 c$ ). These developmental patterns are as expected from the optimal decision array (fig. $3 a$ ), where individuals with a starting belief of 0.5 first traverse the area of state space with more variability in foraging effort before they become more sure of the environment (see example trajectories in app. C). We find similar patterns when predation and food positively covary between environments (fig. A $1 d-\mathrm{A} 1 f$ ) but find little variation over time in the value of information when predation is constant as there is essentially no variation in optimal foraging effort (fig. A $1 a-\mathrm{A} 1 c$ ). Henceforth, we consider only the two scenarios with differential predation to explore information use across development. In the main text, we focus on the scenario with positive covariation between predation and food, while in the appendixes, we present corresponding results for negative covariation.

\section{How Does Starting Belief Affect Phenotypic Development?}

In spite of opportunities for learning during development, there is a lasting effect of having a strong starting belief of
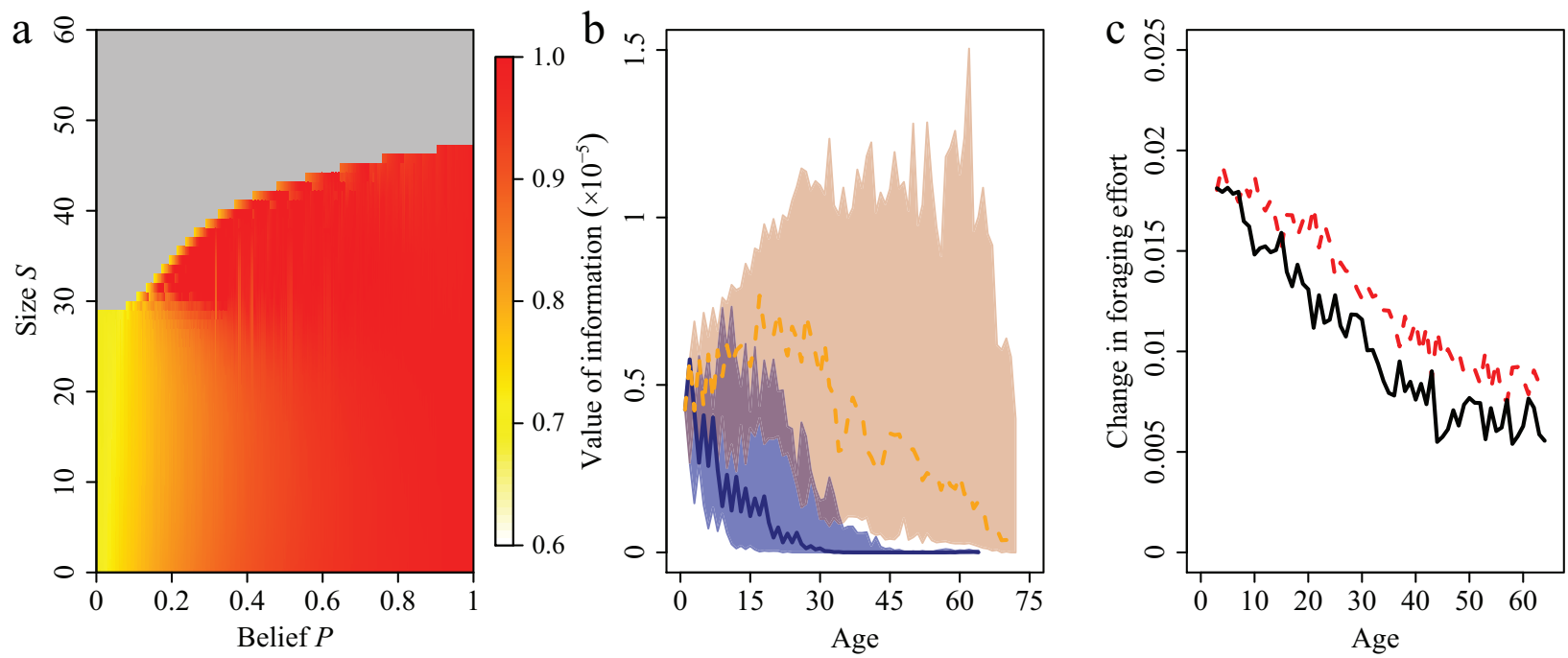

Figure 3: $a$, Optimal foraging effort, $\alpha^{*}$, in relation to information state (belief of being in the high-food environment, $P$ ) and size $(S)$, when the high-food environment also has lower predation. Values are shaded on a spectrum from white $(\alpha=0.6)$ to red $(\alpha=1.0)$, as explained in the legend. Gray indicates states in which the individual should mature. $b$, Age-dependent change in the value of information (median and interquartile range) for 100 individuals developing in the high-food (solid line, blue shading) or low-food (dashed line, orange shading) environment. $c$, Age-dependent change in mean foraging effort (for 200 individuals across both environments) - that is, the extent to which foraging effort varied from one time step to the next-depending on whether an individual found food (black solid line) or did not (red dashed line). Summary data are based on values until the minimum age at maturity in each environment $(b)$ or across both environments $(c)$ so that trends are not obscured by reductions in sample size due to individuals maturing. 
being in the low-food or high-food environment on age and size at maturity compared to individuals who start life with maximal uncertainty ( $P=0.5$; fig. 4$)$. Individuals with a starting belief of 0.1 mature at a later age than the baseline in both environments (fig. $4 a, 4 d$ ), as they adopt a lower foraging effort under the belief that they are in the more dangerous environment (fig. $3 a$ ) and take longer to reach the maturation boundary. The effect of starting belief on size at maturity depends on whether it is matched to the true environment. Individuals with a matched starting belief ( 0.1 in the low-food environment, 0.9 in the high-food environment) mature close to the size that is optimal for that environment, that is, the maturation size of individuals that have perfect knowledge of the environment. A mismatched belief, in contrast, results in individuals maturing too large in the low-food environment and too small in the high-food environment (fig. $4 b, 4 e$ ). These long-term phenotypic effects of the starting belief are weakly reflected in differences in fitness (fig. $4 c, 4 f$ ), with individuals having a mismatched starting belief tending to have lower repro- ductive success than those who start life with an accurate belief about their environment. The long-term effects of starting belief on developmental outcome - in terms of size at maturity and, thus, reproductive success - are similar between the scenario presented here, where high-food environments have low predation and low-food environments have high predation, and the scenario in which levels of food and predation positively covary (fig. A3). The effects on age at maturity differ, however, because individuals with a starting belief of 0.1 mature faster than those with a starting belief of 0.9 , owing to higher optimal foraging effort in the low-predation (and low-food) environment.

\section{What Are the Long-Term Effects of Experiencing Temporary Food Deprivation or Supplementation at Different Stages of Development?}

Heightened sensitivity to the experience of food deprivation and supplementation in early life is evident in terms of age at maturity. Individuals experiencing a period of tem-
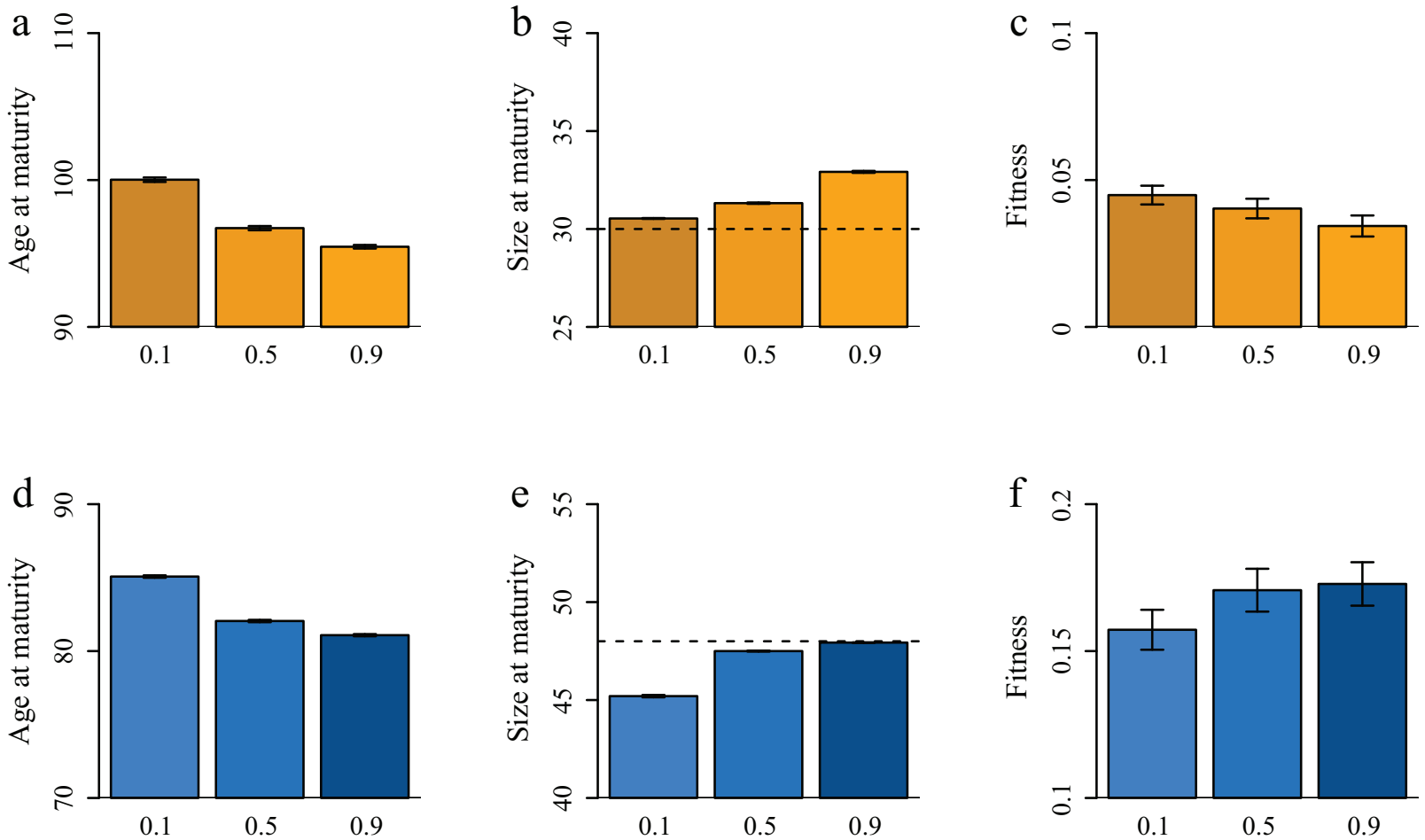

Starting belief $P$

Figure 4: Effect of starting belief on age at maturity $(a, d)$, size at maturity $(b, e)$, and reproductive success $(c, f)$ for individuals in the lowfood (orange, upper panels) and high-food (blue, lower panels) environments. The graphs show mean \pm SE values across 10,000 individuals. A starting belief of 0.5 represents the baseline simulation. Starting beliefs of 0.1 in the low-food environment and 0.9 in the high-food environment are matched to the true environment (shaded darker), whereas starting beliefs of 0.9 in the low-food environment and 0.1 in the high-food environment are mismatched (shaded paler). The horizontal dashed lines in $b$ and $e$ represent the theoretically optimal size at maturity for each environment (for an individual with perfect knowledge). 
porary supplementation mature at an earlier age, whereas those experiencing a period of temporary deprivation delay maturation. There is a stronger effect of deprivation and supplementation when experienced early in life, compared to later in development, for individuals in the low-food environment (fig. $5 a$ ). Similarly, the effect of deprivation is stronger earlier in life for individuals in the high-food environment, although the effect of supplementation does not vary with age (fig. $5 d$ ), presumably because the optimal foraging effort is at its maximum in the high-food environment (fig. 2a). In general, there is no age-dependent sensitivity to deprivation or supplementation in terms of their effects on size at maturity or reproductive success (fig. 5). Individuals experiencing temporary food supplementation mature at a larger size than is optimal in the low-food environment (where optimal refers to the size at which individuals who have perfect knowledge would mature), regardless of whether this experience occurs early or late in development (fig. 5b). Individuals experiencing temporary deprivation mature at a smaller size than is optimal in the high-food environment, again regardless of when this experience occurs. In the high- food environment, individuals have reduced reproductive success when they experience food deprivation and elevated reproductive success when they experience supplementation (fig. 5e) across all time periods. The effect of deprivation or supplementation on reproductive success is not detectable for individuals in the low-food environment (fig. $5 c, 5 f$ ), potentially due to compensatory changes in foraging behavior (fig. C4). We also find age-dependent sensitivity to experiences in terms of age at maturity but not size at maturity or reproductive success, when food availability and predation positively covary (fig. A4). In this case, however, early life experiences have stronger effects than later experiences only when they are mismatched to the prevailing environment (e.g., supplementation in the low-food environment), whereas matched experiences have a stronger effect later in life.

\section{Discussion}

Using a state-dependent model of growth and maturation in an uncertain environment, we have shown that foraging
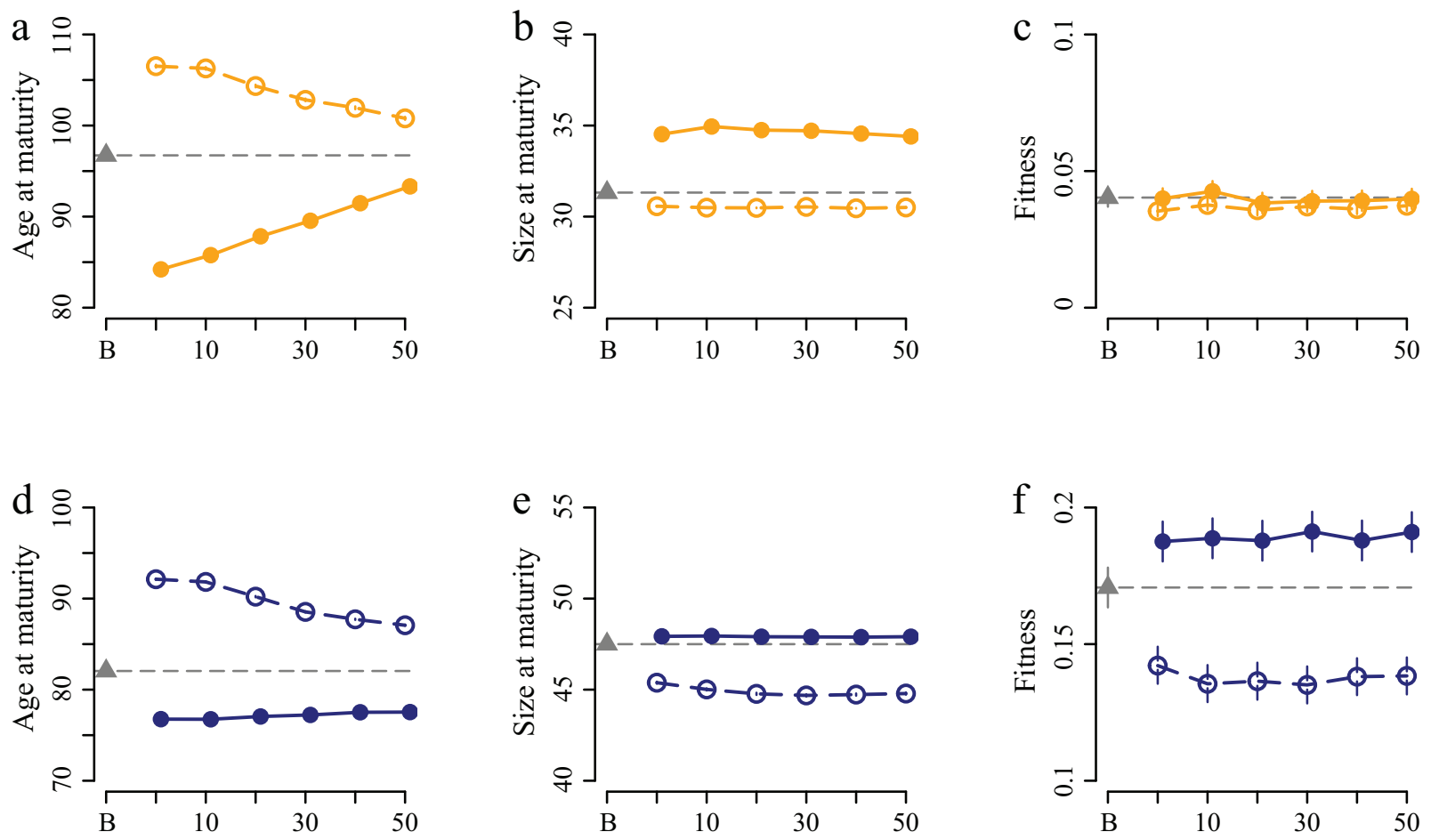

Age at onset of 10-step manipulated foraging experiences

Figure 5: Effect of conditions experienced in specific developmental periods on age at maturity $(a, d)$, size at maturity $(b, e)$, and reproductive success $(c, f)$ for individuals in the low-food (orange, upper panels) and high-food (blue, lower panels) environments. Individuals either experience temporary deprivation (food encountered with probability 0.1 , open circles and dashed lines) or supplementation (food encountered with probability 0.9 , filled circles and lines). Mean \pm SE values across 10,000 individuals are shown (some values are so small that the error bars are not visible), with values from the baseline scenario for comparison (triangle and dashed gray line). Values on the $X$-axis denote age at the start of the 10-step developmental window for each period $(\mathrm{B}=$ baseline). 
behavior is more sensitive to local conditions earlier than later in life for individuals born with maximum uncertainty about their environment. This occurs in tandem with a decline in the value of information with age, as individuals are born uncertain and then gradually learn about their environment. Indeed, this general feature of informational models - that uncertainty is reduced as information is gathered (Mangel 1990) - leads to the expectation that, all else being equal, experiences early in life should have the strongest phenotypic effect (Fawcett and Frankenhuis 2015). Furthermore, our model shows that information at the start of life (akin to inherited genes or maternal effects) can have long-term effects on age and size at maturity. Experiencing temporary food supplementation or deprivation early in life can also affect age at maturity, and mostly (but not always), the effect is stronger than those of experiences later in life. These long-term effects on the phenotype do not always translate into substantial fitness differences, however, because of compensatory changes in behavior. Below, we outline the rationale for these results and discuss their implications for empiricists interested in measuring the longterm effects of early experiences.

Our model shows that both information and phenotypic effects of foraging experiences can result in long-term effects of early experiences without the need for direct costs or constraints on plasticity. An individual experiencing temporary food deprivation early in life, for example, is both smaller in size (phenotypic effect) and more convinced that it is living in the low-food environment (information effect). Most empirical studies on the long-term effects of early life experiences on dispersal, adult size, and reproductive success in wild vertebrates have focused on phenotypic changes and have invoked costs and constraints on plasticity (e.g., Mitchell et al. 2011; Douhard et al. 2013; Lee and Bussière 2013). It would be insightful for empiricists interested in such long-term effects to also measure changes in informational state across development to see whether such long-term effects necessarily require constraints in order to evolve. It is likely that the effect of information gleaned from experiences early in life will be stronger in systems where the pace of environmental change is much longer than the generation time or dispersal distance of individuals and that early life cues are thus reliable indicators of later conditions. It may not be surprising then that the strongest evidence for predictive adaptive responses to early cues has been found in species with a short life cycle, for which individuals in successive generations are likely to encounter similar conditions. In Daphnia, for example, mothers who experience highpredation environments produce offspring with more antipredator defenses (Agrawal et al. 1999), and in the butterfly Bicyclus anynana, individuals who experience early life stress develop a phenotype that incurs a lower cost of dispersal (Saastamoinen et al. 2010). In long-lived species, where early life cues are typically less reliable, adaptive phenotypic responses to such cues are less likely (Hayward et al. 2013; Douhard et al. 2014), and thus constraints may be necessary to explain long-term effects of early experiences.

What is the evidence for early experiences specifically influencing informational state during development? Recent studies have shown that developmental stress influences how animals might acquire information, through effects on exploratory behavior, learning performance, and social learning strategy (Krause et al. 2009; Boogert et al. 2013; Brust et al. 2014; Farine et al. 2015), but these studies have not directly measured the informational state of individuals. We suggest that researchers could attempt to measure their subjects' belief state through changes in their behavior (e.g., foraging effort and mating effort) under different environmental conditions, but this would require some understanding of what the optimal decisions should be in particular conditions. Controlled laboratory observations and learning trials would facilitate such measurements, for example, by following established methods of measuring information acquisition while foraging (Krebs et al. 1978) or more recent social psychology approaches to measuring information use and decision making in humans (Bronfman et al. 2015). Applying these methods to measuring developmental decisions in wild animals may prove more of a challenge, but we believe this could lead to new insights into why sensitive windows occur early in life across a range of natural systems.

Proponents of the developmental programming hypothesis in humans often argue that early life conditions have stronger effects when mismatched to the later environment experienced (Gluckman et al. 2005a), as exemplified by the finding that individuals born during famine conditions have higher risk of coronary and metabolic diseases later in life under improved nutritional conditions (Roseboom et al. 2006). Our model predicts a more complex pattern: the effect of environmental mismatch on the long-term consequences of experiencing a temporary change in food availability depends on the background environment. This environment specificity of long-term effects is in line with several empirical studies that have detected effects of early experiences only in particular adult environments. For example, in preindustrial humans and roe deer (Capreolus capreolus), early life conditions have stronger effects on reproductive success and adult mass, respectively, in adverse adult environments (Rickard et al. 2010; Douhard et al. 2013). In zebra finches (Taeniopygia guttata), the long-term effects of early conditions on immunity are detectable only under favorable adult environments (De Coster et al. 2011), while the effects of developmental stress on later learning and phenotype appear only under harsh conditions (Brust et al. 2014). Many empirical studies measure only the effects of one type of manipulation in one environment (e.g., Kaiser et al. 2003; Careau et al. 2014), and 
we encourage where possible that researchers conduct tests in different contexts (e.g., both experimentally increasing and reducing food availability) under both benign and adverse conditions.

Our model also predicts that experiences in early life will not always exert stronger effects than those later in life. Indeed, when food and predation positively covary, experiences matched to the environment have stronger effects when they occur later in development (fig. A4). It is thus important that tests of early life conditions repeat the same treatment at different stages of development to demonstrate unequivocally the existence of sensitive windows, rather than focusing exclusively on an early stage of development as in most previous studies (e.g., Kuzawa et al. 2010; Douhard et al. 2013; but see Hopwood et al. 2013; Wong and Kölliker 2014).

Although our model predicts long-term effects of information at the start of life and experiences during development on later-life phenotype, these do not translate into measurable effects on fitness. This is likely due to robustness in the system: through state-dependent behavior, individuals are continually adjusting their phenotype to maximize their expected reproductive success given local conditions (fig. 1). By adjusting their foraging effort and the age at which they mature, individuals can modify their age-specific survival and size at maturity to mitigate the effects of changes in the environment. Detrimental effects of early life conditions in our simulations were small because of the shape of the fitness landscape, which enables different life-history strategies to achieve similar reproductive success. Thus, observations of long-term effects of early environmental conditions may not necessarily be associated with detectable fitness outcomes and, hence, may have limited impact at the population level even if they change the life-history trajectories of individuals. This caveat may be of interest to those studying the link between early development and the onset of metabolic disease in humans, where an effect on health is not necessarily the same as an effect on fitness even though the term "maladaptive" is often used for these effects (Gluckman et al. 2007).

We found that the value of information and sensitivity of behavior to experience declines with age, which is consistent with recent theoretical studies relating information use to patch choice (Lister 2014) and boldness (Stamps and Krishnan 2014) across development. Here, in common with several other models of adaptive developmental plasticity (e.g., Frankenhuis and Panchanathan 2011a; Stamps and Krishnan 2014; but see Fischer et al. 2014), we assumed that the environment is unchanging over development (apart from our simulations of temporary food supplementation or deprivation). Our model could be extended to allow for local conditions potentially changing during development; for example, because the environment varies temporally (as is typical in many Bayesian models of learning during foraging; McNamara and Houston 1987; McNamara 1996; Tenhumberg et al. 2000; Eliassen et al. 2007) or individuals disperse during development. Environmental heterogeneity during development may weaken the long-term effects of early life experiences on age at maturity and may result in the value of information remaining high across development. It is also possible that adaptation to fluctuating environmental conditions increases the magnitude of effects of information use on behavior, as individuals should exploit good periods if they are not likely to persist (Higginson et al. 2012).

Our model highlights how understanding the evolution of sensitive windows during growth requires an appreciation of the coupled dynamics of information updating and phenotypic change (Stamps and Krishnan 2014). We have presented a theoretical framework to understand these dynamics and have suggested the types of empirical studies that can test our predictions. As well as modeling these coupled dynamics, there are several key differences between our model and previous models on information use during development (Frankenhuis and Panchanathan 2011a; Fischer et al. 2014; Lister 2014). We assumed directional selection for larger size, regardless of the environmental conditions, and that phenotypic reversal (i.e., reduction in size) is not possible. This is in contrast to the focus on divergent specialization in previous models, where development toward the optimum phenotype for one environment implies either a less appropriate phenotype for the other environment (Fischer et al. 2014) or less opportunity to develop the appropriate phenotype for the other environment (Frankenhuis and Panchanathan 2011a; Fischer et al. 2014). Longterm effects of early life conditions readily arise when alternative phenotypes diverge in this way (Frankenhuis and Panchanathan 2011a; Fischer at al. 2014), but our model shows that similar effects can arise even when a larger body size is always preferable. We also allowed the timing of maturation to be flexible, in contrast to previous models that imposed a fixed end to development (Frankenhuis and Panchanathan 2011a; Fischer et al. 2014; Lister 2014). In the model of incremental development by Frankenhuis and Panchanathan (2011a), the assumption of fixed developmental time resulted in opportunity costs, whereas in the case of unlimited plasticity modeled by Fischer et al. (2014), phenotypic adjustments directly reduced survival or fecundity. In contrast, we showed that heightened sensitivity to experiences early in life can evolve in the absence of any such constraints on plasticity.

Our model assumptions make it most applicable to cases of adaptive developmental plasticity under an informational framework (Nettle and Bateson 2015): individuals in the model respond to cues and can continually adjust their phenotype, unlike in the somatic-state-based framework whereby phenotypic adjustments are irreversible (as in some 
previous models). We hope that our model will provide an insightful starting point for future models with increased complexity, tailored to specific systems. Future models could, for instance, consider explicit physiological constraints that may impose costs of adverse early conditions, such as lagged costs of rapid early growth through accumulation of cellular damage (Metcalfe and Monaghan 2003). As we state above, many empirical examples of early life experiences - particularly in long-lived species - are likely due to such physiological constraints. Our assumption that reproductive success is a function of size at maturity may mean that our predictions may not hold for those species with indeterminate growth; and we did not allow individuals to disperse away from poor patches, which might be the case in highly mobile species. Future developments could study the whole life history, although since there will be a positive effect of size at maturity even in organisms with indeterminate growth, we do not expect dramatically different results.

In summary, we have used a general model of biological growth with flexible timing of maturity to show that early life experiences can sometimes influence later life history even when there are no constraints on plasticity. There is growing appreciation that experience at particular times of life-often early in development-have striking effects on behavior, health, and reproductive success yet only recently have theoretical models attempted to explicitly address the question of how such effects might evolve. Our model shows that understanding how animals acquire and use information during development can lead to insights on why experiences at particular stages have strong effects on development. While these informational effects may be more challenging to measure in empirical studies than direct phenotypic changes, considering the availability and reliability of cues may lead to a better understanding of how sensitive windows have evolved, why they are more pronounced in certain systems, and whether they will only be detected under particular adult environments.

\section{Acknowledgments}

We thank M. Douhard, W. Frankenhuis, J.-M. Gaillard, M. Mangel, N. Metcalfe, and J. Stamps for insightful comments on an earlier version of the article. This research was funded by the European Union's Seventh Framework Programme (FP7/2007-2011) under grant 259679 (IDEAL) awarded to T.U. T.W.F., A.D.H., and P.C.T. were supported by the European Research Council (ERC Advanced Grant 250209 Evomech to A. Houston). T.U. was supported by the Royal Society of London and the Knut and Alice Wallenberg Foundation. A.D.H. was supported by fellowships from the Wissenschaftskolleg zu Berlin and the Natural Environment Research Council (grant NE/L011921/1).

\section{Literature Cited}

Agrawal, A. A., C. Laforsch, and R. Tollrian. 1999. Transgenerational induction of defences in animals and plants. Nature 401:6063.

Albon, S. D., T. H. Clutton-Brock, and F. E. Guinness. 1987. Early development and population dynamics in red deer. II. Densityindependent effects and cohort variation. Journal of Animal Ecology 56:69-81.

Bateson, P. 1979. How do sensitive periods arise and what are they for? Animal Behaviour 27:470-486.

Bateson, P. P. G. 1966. The characteristics and context of imprinting. Biological Reviews 41:177-211.

Boogert, N., C. Zimmer, and K. Spencer. 2013. Pre- and post-natal stress have opposing effects on social information use. Biology Letters 9:20121088.

Bronfman, Z. Z., N. Brezis, R. Moran, K. Tsetsos, T. Donner, and M. Usher. 2015. Decisions reduce sensitivity to subsequent information. Proceedings of the Roval Societv B: Biological Sciences 282: 20150228.

Brust, V., O. Krüger, M. Naguib, and E. T. Krause. 2014. Lifelong consequences of early nutritional conditions on learning performance in zebra finches (Taeniopygia guttata). Behavioural Processes 103: 320-326.

Burgess, S. C., and D. J. Marshall. 2014. Adaptive parental effects: the importance of estimating environmental predictability and offspring fitness appropriately. Oikos 123:769-776.

Cam, E., J.-Y. Monnat, and J. E. Hines. 2003. Long-term fitness consequences of early conditions in the kittiwake. Iournal of Animal Ecology 72:411-424

Careau, V., W. A. Buttemer, and K. L. Buchanan. 2014. Early developmental stress, repeatability, and canalization in a suite of physiological and behavioral traits in female zebra finches. Integrative and Comparative Biology 54:539-554.

Case, T. J. 1978. On the evolution and adaptive significance of postnatal growth rates in the terrestrial vertebrates. Quarterly Review of Biology 53:243-282.

Clark, C. W., and M. Mangel. 2000. Dynamic state variables in ecology: methods and applications. Oxford University Press, New York.

De Coster, G., S. Verhulst, E. Koetsier, L. De Neve, M. Briga, and L. Lens. 2011. Effects of early developmental conditions on innate immunity are only evident under favourable adult conditions in zebra finches. Naturwissenschaften 98:1049-1056.

Douhard, M., J.-M. Gaillard, D. Delorme, G. Capron, P. Duncan, F. Klein, and C. Bonenfant. 2013. Variation in adult body mass of roe deer: early environmental conditions influence early and late body growth of females. Ecology 94:1805-1814.

Douhard, M., F. Plard, J.-M. Gaillard, G. Capron, D. Delorme, F. Klein, P. Duncan, L. E. Loe, and C. Bonenfant. 2014. Fitness consequences of environmental conditions at different life stages in a long-lived vertebrate. Proceedings of the Roval Societv B: Biological Sciences 281:20140276.

Dufty, A. M., J. Clobert, and A. P. Møller. 2002. Hormones, developmental plasticity and adaptation. Trends in Ecology and Evolution 17:190-196.

Eliassen, S., C. Jørgensen, M. Mangel, and J. Giske. 2007. Exploration or exploitation: life expectancy changes the value of learning in foraging strategies. Oikos 116:513-523.

Ellis, B. J., W. T. Boyce, J. Belsky, M. J. Bakermans-Kranenburg, and M. H. van Ijzendoorn. 2011. Differential susceptibility to the en- 
vironment: an evolutionary-neurodevelopmental theory. Development and Psychopathology 23:7-28.

English, S. 2015. Code and simulation data from: Adaptive use of information during growth can explain long-term effects of early life experiences. American Naturalist, Github Repository, http://dx.do .org/10.5281/zenodo.35322.

English, S., I. Pen, N. Shea, and T. Uller. 2015. The information value of non-genetic inheritance in plants and animals. PLoS ONE 10 e0116996.

Farine, D. R., K. A. Spencer, and N. J. Boogert. 2015. Early-life stress triggers juvenile zebra finches to switch social learning strategies. Current Biology 25:2184-2188.

Fawcett, T. W., and W. E. Frankenhuis. 2015. Adaptive explanations for sensitive windows in development. Frontiers in Zoology 12:S3.

Fischer, B., G. S. van Doorn, U. Dieckmann, and B. Taborsky. 2014. The evolution of age-dependent plasticity. American Naturalist 183:108-125.

Frankenhuis, W. E., and K. Panchanathan. 2011a. Balancing sampling and specialization: an adaptationist model of incremental development. Proceedings of the Roval Society B: Biological Sciences 278 $3558-3565$.

- 2011b. Individual differences in developmental plasticity may result from stochastic sampling. Perspectives on Psychological Science 6:336-347.

Frankenhuis, W. E., K. Panchanathan, and H. Clark Barrett. 2013. Bridging developmental systems theory and evolutionary psychology using dynamic optimization. Developmental Science 16:584598.

Gluckman, P. D., M. A. Hanson, and A. S. Beedle. 2007. Non-genomic transgenerational inheritance of disease risk. BioEssavs 29:145-154

Gluckman, P. D., M. A. Hanson, and H. G. Spencer. 2005a. Predictive adaptive responses and human evolution. Trends in Ecology and Evolution 20:527-533.

Gluckman, P. D., M. A. Hanson, H. G. Spencer, and P. Bateson. 20056. Environmental influences during development and their later consequences for health and disease: implications for the interpretation of empirical studies. Proceedings of the Roval Societv B: Biological Sciences 272:671-677.

Harrison, X. A., J. D. Blount, R. Inger, D. R. Norris, and S. Bearhop. 2011. Carry-over effects as drivers of fitness differences in animals. Iournal of Animal Ecology 80:4-18.

Hayward, A. D., I. J. Rickard, and V. Lummaa. 2013. Influence of early-life nutrition on mortality and reproductive success during a subsequent famine in a preindustrial population. Proceedings of the National Academy of Sciences of the USA 110:13886-13891.

Higginson, A. D., T. W. Fawcett, P. C. Trimmer, J. M. McNamara, and A. I. Houston. 2012. Generalized optimal risk allocation: foraging and antipredator behavior in a fluctuating environment. American Naturalist 180:589-603.

Hilborn, R., and M. Mangel. 1997. The ecological detective: confronting models with data. Princeton University Press, Princeton, NJ.

Hopwood, P. E., A. J. Moore, and N. J. Royle. 2013. Nutrition during sexual maturation affects competitive ability but not reproductive productivity in burying beetles. Functional Ecology 27:13501357.

Houston, A. I., and J. M. McNamara. 1989. The value of food: effects of open and closed economies. Animal Behaviour 37:546-562.

- 1999. Models of adaptive behaviour: an approach based on state. Cambridge University Press, Cambridge.
Hoverman, J. T., and R. A. Relyea. 2007. How flexible is phenotypic plasticity? developmental windows for trait induction and reversal. Ecology 88:693-705.

Jönsson, K. I. 1997. Capital and income breeding as alternative tactics of resource use in reproduction. Oikos 78:57-66.

Kaiser, S., F. P. M. Kruijver, D. F. Swaab, and N. Sachser. 2003. Early social stress in female guinea pigs induces a masculinization of adult behavior and corresponding changes in brain and neuroendocrine function. Behavioural Brain Research 144:199-210.

Kaiser, S., and N. Sachser. 2005. The effects of prenatal social stress on behaviour: mechanisms and function. Neuroscience and Biobehavioral Reviews 29:283-294.

Krause, E. T., M. Honarmand, J. Wetzel, and M. Naguib. 2009. Early fasting is long lasting: differences in early nutritional conditions reappear under stressful conditions in adult female zebra finches. PLoS ONE 4:e5015.

Krebs, J. R., A. Kacelnik, and P. Taylor. 1978. Test of optimal sampling by foraging great tits. Nature 275:27-31.

Kuzawa, C. W., T. W. McDade, L. S. Adair, and N. Lee. 2010. Rapid weight gain after birth predicts life history and reproductive strategy in Filipino males. Proceedings of the National Academv of Sciences of the USA 107:16800-16805.

Lee, P., and L. Bussière. 2013. Enduring consequences of early experiences: 40 year effects on survival and success among African elephants (Loxodonta africana). Biology Letters 9:20130011.

Lee, W., P. Monaghan, N. B. Metcalfe, A. Health, and G. K. Building. 2012. The pattern of early growth trajectories affects adult breeding performance. Ecology 93:901-912.

Leimar, O., P. Hammerstein, and T. J. M. Van Dooren. 2006. A new perspective on developmental plasticity and the principles of adaptive morph determination. American Naturalist 167:367-376.

Lindström, J. 1999. Early development and fitness in birds and mammals. Trends in Ecology and Evolution 14:343-348.

Lister, B. C. 2014. Information, behaviour and population dynamics. Oikos 123:1431-1438.

Lucas, A. 1998. Programming by early nutrition: an experimental approach. Journal of Nutrition 128:401S-406S.

Mangel, M. 1990. Dynamic information in uncertain and changing worlds. Journal of Theoretical Biology 146:317-332.

Mangel, M., and B. D. Roitberg. 1989. Dynamic information and host acceptance by a tephritid fruit fly. Ecological Entomology 14:181189.

McNamara, J. M. 1996. Risk-prone behaviour under rules which have evolved in a changing environment. American Zoologist 36:484495.

McNamara, J. M., and S. R. X. Dall. 2010. Information is a fitness enhancing resource. Oikos 119:231-236.

McNamara, J. M., R. F. Green, and O. Olsson. 2006. Bayes' theorem and its applications in animal behaviour. Oikos 112:243-251.

McNamara, J. M., and A. I. Houston. 1987. Memory and the efficient use of information. Lournal of Theoretical Biology 125:385-395.

Metcalfe, N. B., F. A. Huntingford, and J. E. Thorpe. 1988. Feeding intensity, growth rates, and the establishment of life-history patterns in juvenile Atlantic salmon Salmo salar. 57:463-474.

Metcalfe, N. B., and P. Monaghan. 2003. Growth versus lifespan: perspectives from evolutionary ecology. Experimental Gerontology 38:935-940.

Mitchell, G. W., C. G. Guglielmo, N. T. Wheelwright, C. R. FreemanGallant, and D. R. Norris. 2011. Early life events carry over to influ- 
ence pre-migratory condition in a free-living songbird. PLoS ONE 6:e28838.

Nettle, D., and M. Bateson. 2015. Adaptive developmental plasticity: what is it, how can we recognize it and when can it evolve? Proceedings of the Roval Societv B: Biological Sciences 282:20151005.

Plard, F., N. G. Yoccoz, C. Bonenfant, F. Klein, C. Warnant, and J.-M. Gaillard. 2015. Disentangling direct and growth-mediated influences on early survival: a mechanistic approach. Iournal of Animal Ecology 84:1363-1372.

R Development Core Team. 2013. R: a language and environment for statistical computing. R Foundation for Statistical Computing, Vienna. http://www.R-project.org.

Rickard, I. J., J. Holopainen, S. Helama, S. Helle, A. F. Russell, and V. Lummaa. 2010. Food availability at birth limited reproductive success in historical humans. Ecology 91:3515-3525.

Roseboom, T., S. de Rooij, and R. Painter. 2006. The Dutch famine and its long-term consequences for adult health. Early Human Development 82:485-491.

Saastamoinen, M., D. van der Sterren, N. Vastenhout, B. J. Zwaan, and P. M. Brakefield. 2010. Predictive adaptive responses: conditiondependent impact of adult nutrition and flight in the tropical butterfly Bicyclus anynana. American Naturalist 176:686-698.

Satterthwaite, W. H., M. P. Beakes, E. M. Collins, D. R. Swank, J. E. Merz, R. G. Titus, S. M. Sogard, and M. Mangel. 2009. Steelhead life history on California's central coast: insights from a state-dependent model. Transactions of the American Fisheries Society 138:532-548.

2010. State-dependent life history models in a changing (and regulated) environment: steelhead in the California Central Valley. Evolutionarv Applications 3:221-243.

Shea, N., I. Pen, and T. Uller. 2011. Three epigenetic information channels and their different roles in evolution. Iournal of Evolutionarv Biology 24:1178-1187.

Stamps, J. A., and V. V. Krishnan. 2014. Combining information from ancestors and personal experiences to predict individual differences in developmental trajectories. American Naturalist 184:647-657.

Tenhumberg, B., A. J. Tyre, and B. Roitberg. 2000. Stochastic variation in food availability influences weight and age at maturity. Journal of Theoretical Biology 202:257-272.
Thorpe, J. E., M. Mangel, N. B. Metcalfe, and F. A. Huntingford. 1998. Modelling the proximate basis of salmonid life-history variation, with application to Atlantic salmon, Salmo salar L. Evolutionary Ecology 12:581-599.

Trimmer, P. C., A. I. Houston, J. A. R. Marshall, M. T. Mendl, E. S. Paul, and J. M. McNamara. 2011. Decision-making under uncertainty: biases and Bayesians. Animal Cognition 14:465-476.

Uller, T. 2008. Developmental plasticity and the evolution of parental effects. Trends in Ecology and Evolution 23:432-438.

Valone, T. 2006. Are animals capable of Bayesian updating? an empirical review. Oikos 112:252-259.

Welton, N. J., J. M. McNamara, and A. I. Houston. 2003. Assessing predation risk: optimal behaviour and rules of thumb. Theoretical Population Biology 64:417-430.

Werner, E. E. 1986. Amphibian metamorphosis: growth rate, predation risk, and the optimal size at transformation. American Naturalist 128:319-341.

Werner, E. E., and J. F. Gilliam. 1984. The ontogenetic niche and species interactions in size-structured populations. Annual Review of Ecology and Systematics 15:393-425.

West-Eberhard, M. J. 2003. Developmental plasticity and evolution. Oxford University Press, Oxford.

Whitman, D. W., and A. A. Agrawal. 2009. What is phenotypic plasticity and why is it important? Pages 1-63 in D. W. Whitman and T. N. Ananthakrishnan, eds. Phenotypic plasticity of insects: mechanisms and consequences. Science Publishers, Enfield.

Wong, J. W. Y., and M. Kölliker. 2014. Effects of food restriction across stages of juvenile and early adult development on body weight, survival and adult life history. Iournal of Evolutionarv Biology 27:2420-2430.

Worthman, C. M., and J. Kuzara. 2005. Life history and the early origins of health differentials. American Journal of Human Biology 17:95112.

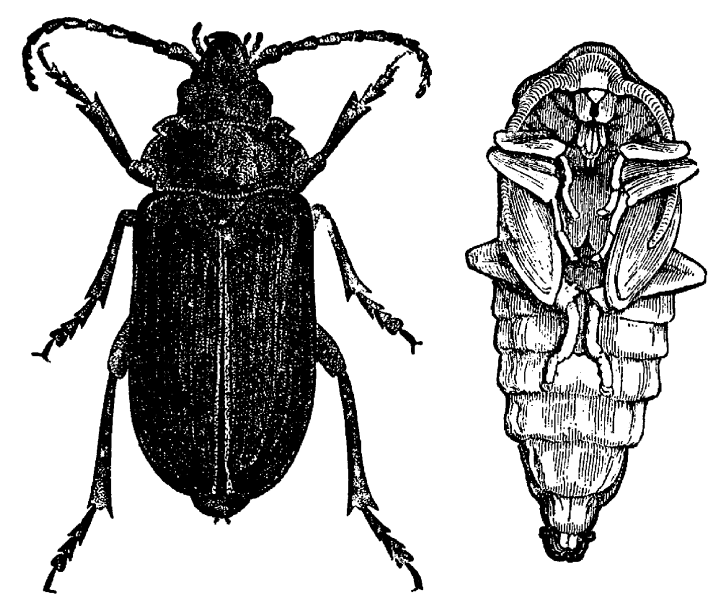

Associate Editor: Jean-Michel Gaillard Editor: Judith L. Bronstein

"The grub ... has, with that of the Broad-necked Prionus (P. laticollis of Drury [illustrated]), as Harris states, 'almost entirely destroyed the Lombardy poplar in this vicinity (Boston). It bores in the trunks, and the beetle flies by night in August and September." From "The Borers of Certain Shade Trees" by A. S. Packard Jr. (The American Naturalist, 1870, 4:588-594). 